\title{
Ceil' İdentity
}

\section{Snapshot - changing melanocyte identity in melanoma developing route}

\author{
Monica Neagu ${ }^{1,2,3}$, Carolina Constantin ${ }^{1,3}$, Ayse Basak Engin ${ }^{4}$, Iulia Popescu ${ }^{5}$ \\ 1 Immunology Laboratory, „Victor Babes” National Institute of Pathology, \\ Bucharest, Romania \\ 2 Doctoral School, Biology Faculty, University of Bucharest, Romania \\ 3 Pathology Department, Colentina Clinical Hospital, Bucharest, Romania \\ 4 Department of Toxicology, Faculty of Pharmacy Gazi University, Ankara, Turkey \\ 5 Division of Pulmonary, Allergy and Critical Care Medicine, Department of Medicine, \\ School of Medicine, University of Pittsburgh, Pittsburgh, Pennsylvania, USA
}

\begin{abstract}
The largest organ with immune function, the skin, has complex structure and various physiological functions. Cells comprising this complex structure sustain various processes and have proteomic/transcriptomic/genomic patterns that would subside to developing a specific function in a specific moment of time and in a defined space. Within the complex skin structure melanocyte is one of the cell types that is involved in skin's main functions. In the process of normal melanocyte transformation into a neoplastic cell there are several stages that are favored by a protumor inflammatory milieu. A tumorigenesis-friendly environment would increase cell's genetic instability that will further lead to tumorigenesis and additionally to metastasis. In the environment, immune cells and immune-related molecules seminally contribute to the inflammatory landscape. Melanomagenesis is not a straight forward process. In order to take place, various factors need to collide, environmental, genetic, and immune factors must conjoint. Melanomas are heterogeneous and the transformed melanocyte has various genetic alterations, these mutations being specific to the site, to the degree of UV exposure, and/or specific for the genetic make-up of the host's organism. This variability suggests that melanoma has more than one causal pathway. Within our paper we will snapshot the cellular identity of normal melanocyte, through benign transformed melanocyte up to a full blown tumorigenesis. Factors that are triggering these transformations(s) will be briefly highlighted.
\end{abstract}

Key words:

melanoma, melanomagenesis, inflammation, cell identity

\section{Article Info}

Received 13 May 2020

Accepted 29 June 2020

Available online 20 October 2020

DOI

10:47570/joci.2020.003

JoCl year 1, vol. 1, p. $0033-0047$, October, 20, 2020

Corresponding author:

neagu.monica@gmail.com 


\section{Introduction}

Tumors and their specific microenvironment have a complex structure in terms of cells and factors. This complex architecture is composed of various cell types, cell (sub)populations including tumor cells, stromal and immune cells. The complexity of tumors is increased by the fact that during its development each cell type has sub-populations that are heterogenic; hence there are different clones of both tumor cells and infiltrated immune cells (limenez-Sanchez et al., 2017; McGranahan and Swanton, 2017).

These different cell types interact and communicate via secreted molecules and/or via ligand-receptor interactions. One type of ligand can be secreted and bind to its proximity receptor and/or can be anchored in the cellular membrane so that the actual cellular partners will physically interact (Ramilowski et al. 2015). These interactions, either direct or indirect, mediated by soluble secreted molecules would alter cells genomic, proteomic, transcriptomic and metabolomic patterns. These cellular profiles would finally govern several important processes like tumorigenesis, tumor progression, therapy resistance installment, immune infiltration and overall inflammatory processes (Hanahan and Weinberg, 2011). Skin melanoma follows all the mentioned traits. Actually, in this type of tumor, the first immune therapy that directly targeted cells' interaction was studied and then implemented in clinics. Therefore, the immune checkpoint inhibitors are targeting the cellular interaction CD28-CTLA4 (ipilimumab), or are targeting the PD1-PDL1 interaction (pembrolizumab, nivolumab) (Pardoll, 2012). After being approved in melanoma, these ligand-based therapies were approved in several other cancers, but the clinical response is positive only in up to $25 \%$ of the patients (Dempke et al., 2017; Schumacher et al., 2015). It is obvious that these overall limited responses to immuno-drugs are due to the complex mechanisms and intricate network of cell-cell interactions (Sarkar et al., 2016). Discovering additional cellular interactions to be targeted, identifying new traits of tumor microenvironment and deciphering molecular patterns of cells residing in tumor microenvironment would enhance knowledge and would lead to new therapy targets (Kumar et al., 2018).

\section{Normal Melanocyte cellular identity}

Melanoblasts that are derived from the neural crest are undifferentiated and non-pigmented cells that, when they migrate to the epidermis, will start to synthesize melanin in melanosomes and would transform into adult melanocytes.

Through-out their normal development, melanocytes identity is characterized by Pax3, Sox10, endothelin3 (ED-3), its specific receptor

(Endrb), c-Kit and Mitf expression. Upon synthesis by melanocytes, melanin is transferred to keratinocytes to protect the skin against ultraviolet radiation (UV). In specialized organelles, melanosomes, through specialized enzymatic apparatus (tyrosinase, tyrosinase-related protein-1 - TYRP1, tyrosinase-related protein 2/dopachrome tautomerase - DCT) two types of melanin are formed. Eumelanin (brown/black pigment) has photoprotective potency and pheomelanin (orange/yellow pigment) has lower photoprotective properties. Genetic, environmental and endocrine factors determine the amount, type, and distribution of melanins in the skin, hair, and eyes.

p53 activation in keratinocytes upon ultraviolet irradiation (UVR) induces proopiomelanocortin (POMC) transcription, precursor for

-melanocyte stimulating hormone ( $\mathrm{MSH}$ ) or adrenocorticotropic hormone (ACTH), hormones that have pro-pigmenting properties (Cuit et al., 2007). MSH activates melanocortin-1receptor (MC1R) and further activates CAMP/protein kinase $A$ (PKA)/CREB signaling pathway increasing the Microphthalmia-associated transcription factor (MITF) (Abdel-Malek et al., 2000; Bertolotto et al. 1998). MITF, a key marker for melanocytes, is directly linked to pigment production and melanin synthesis (Gaggioli et al., 2003). MITF regulates genes that induce melanosome biogenesis (Hoek et al., 2008; Vetrini et al., 2004), melanin synthesis (Bertolotto et al., 1996, Bertolotto et al., 1998), and melanosome trafficking (Chiaverini et al., 2008, Passeron et al., 2004). Thus, MITF expression is an important identification marker for an active melanocyte as it regulates important cellular responses, mainly sustaining the photoprotection of the skin (Van Schanke et al., 2005, Walker et al., 2009).

Keratinocyte release several factors (e.g. $\mathrm{MSH}$, endothelin, granulocyte-macrophage colony-stimulating factor - GM-CSF, leukemia in- 
hibitory factor - LIF, basic fibroblast growth factor - bFGF, hepatocyte growth factor - HGF) that induce melanocyte proliferation (Hirobe, 2005). All these molecules activate their specific receptors activating MAPK/ERK and $\mathrm{PI} 3 \mathrm{~K} / \mathrm{AKT}$ signaling pathways. Twenty years ago, the first proof of $\mathrm{v}$-Raf murine sarcoma viral oncogene homolog B1 (BRAF) expression and its involvement in melanocyte function was reported. The study showed that BRAF activation via the

MSH/CAMP pathway increases proliferation via MEK/ERK signaling pathway (Busca et al, 2000). Actually, this finding opened the field of research and further expanded the clinical applications focusing on the role of BRAF in skin melanoma (Bertolotto 2013). ERK and $\mathrm{Pl}_{3} \mathrm{~K}$ signaling pathways are involved in the melanocytes function of photoprotection against UV. These cells have anti-apoptotic mechanisms resisting to the UV-induced DNA damage. Upon UV, MITF expression is increased and several anti-apoptotic genes expression are involved, such as BCL2 (McGill et al., 2002), BCL2A1 (Haqa et al., 2013), ML-IAP (Melanoma inhibitor of apoptosis protein) (Dynek et al., 2008) along with the up-regulation of genes involved in DNA repair (Strub et al., 2011).

\section{Melanocytes comprised in benign nevi}

The cell identity of melanocytes within melanocytic nevi has particularities. The majority of human, congenital melanocytic nevi have RASQ61K/R mutations and the majority of acquired nevi have BRAFV60oE mutation (Dadras, 2011). When oncogenic NRAS or BRAF are induced to be over-expressed in normal melanocytes a senescence phenotype is triggered (Denoyelle et al., 2006, Michaloglou et al., 2005), thus proving that nevi have an oncogene-induced senescence. Melanocytes within the nevi can have the cellular growth arrested for tens of years.

In animal experimental models that express NRASQ61K (Ackermann et al., 2005) or BRAFV60oE (Dankort et al., 2009, Dhomen et al., 2009) it was shown that benign melanocytic nevi develop, these nevi rarely progressing to melanoma. But if several regulatory proteins are missing, the mouse model will develop in an accelerated pace melanomas. Therefore lacking p16INK4a (Ackermann et al, 2015), Pten (Dankort et al, 2009), or -catenin (Damsky et al.,
2015), melanomagenesis will be accelerated. Similarly, in humans various genetic/epigenetic modifications along with extrinsic stimuli would drive melanoma development (Whiteman et al., 2011).

\section{Extracellular settings favoring melanomagenesis}

\section{Inflammation Environment}

In the process of normal melanocyte transformation into a neoplastic cell there are several stages that are favored by a pro-inflammatory milieu. A tumor-friendly environment would increase cell's genetic instability that would lead to tumorigenesis and further to metastasis. In the tumor microenvironment (TME) immune cells and immune related molecules seminally contribute to the inflammatory landscape. Although the most studied cellular interface within the tumor is the tandem tumor cell - cytotoxic T cell (CTLs), presently the involvement of other non-immune and immune cells have been largely recognized. Therefore, other immune cells can contribute to tumor development (Barnes and Amir, 2017) and when predicting clinical evolution of melanoma patients the inflammatory immune cells can prognosticate high risk recurrence (Weiss et al., 2016). Immune cells proportion and types establish the inflammatory pattern of the tumor and characterizes the balance between an efficient local antitumor response and a pro tumor milieu (Zurac et al., 2013; Neagu(a) et al., 2019).

Immune cells in the context of anti-tumor action are subjected to three stages: elimination (cancer immunosurveillance), equilibrium, and escape (Dunn et al., 2004). Notably, the entire newly created TME contributes to establishing an immunosuppressive network (Neagu(a) et al, 2015). In this network, stromal cells interrelate with inflammatory immune cells and vascular system cells. The molecular pattern of TME abounds in molecules like cytokines, chemokines and growth factors that sustain the tumor immune escape (Karlou et al., 2015; Vinay et al., 2015).

Overcoming tumorigenesis and/or responding to therapy are processes dependent on TME particularities. There are two distinct mechanisms of resistance: one is sustained by the existence of immune-suppressive cells and the 
other is sustained by the lack of active immune cells. When tumors have a high infiltration of CD8 ${ }^{+}$CTLs, B cells and macrophages an immune-suppressive cellular milieu is established where the immune resistance process is sustained by negative immuneregulators. In non-inflammed tumors the absence of T-cells and innate immunity regulators sustain the resistance. Therefore, although the main process is therapy resistance, the particularities of immune cells are different and consequently the means to overcome this resistance would be different (Gajewski et al., 2017; Spranger, 2011). NK cells that infiltrate melanoma tumors can induce in the TME epithelial-to-mesenchymal transition (EMT) processes inducing thus a more aggressive tumor cell phenotype. It was shown that NKp3o or NKG2D receptors and IFNY combined with TNFa release are involved in this process. Melanoma cells subjected to EMT would increase HLA-I surface expression or would inhibit specific receptors that trigger NK activation, evading NK cells attack. This process can be further exploited in new anti-tumour therapies based on manipulating NK cells in melanoma (Huergo-Zapico et al., 2018; Neagu(b) et al, 2019). The capacity of tumor cells to escape the immune system's action has been suggested as a "hallmark of cancer" (Hanahan and Weinberg RA, 2011).

The skin is a complex tissue, actually it is the biggest organ with immune function (Neagu, 2012) and within this organ there is a clear interaction between tissue damage, inflammation and cancer development. Skin is subjected to various environmental aggressors and in physiological conditions the inflammatory reactions generated by these aggressors have to fulfill a self-limiting process (Neagu(a) et al, 2019). The tumorigenesis process that can affect besides melanocytes, other resident cells as well, can be triggered by constitutive pathway activation (Gonda et al., 2009). Inflammation can have acute and/or chronic stages, and these stages can tilt the physiological balance toward skin's regeneration or toward skin's tumorigenesis.

If the inflammatory conditions are maintained a chronic inflammation will appear, this condition favoring precancerous lesion (Castle et al., 2001; Rothenberg and Ellisen, 2012; Arzumanyan et al., 2013, Salama et al., 2013, Houghton, 2013). This pre-cancerous lesion if attacked by an active immune response would subside to healing and the melanocyte will return to normal patterns. But if chronic inflammation is installed the process can lead to primary tumor development.

Therefore, a robust immune system can recognize, detect and destroys early many tumor cells, but when immune-suppression appears the tumor will expand and become clinically apparent (Dunn et al., 2004, Raval et al., 2014). We have shown in melanoma patients that a chronic inflammation, long-term production and accumulation of inflammatory factors (e.g. cytokines/chemokines) can induce both locally and systemically an immunosuppressant milieu associated with cancer progression (Neagu et al., 2013).

Fifteen years ago, probably one of the first studies, it was shown that in primary melanoma tumors CXCR4 expression is associated with tumor ulceration, tumor thickness and clinically higher mortality rate (Longo-Imedio et al., 2005). Later on, it was shown that CXCR3 is expressed on invasive skin melanomas associated with tumor thickness $>1 \mathrm{~mm}$ (Monteagudo et al., 2007).

It is important to note, that in the metastatic process chemokines and their specific receptors are the molecules seminally involved in organ selectivity. Through chemokine-receptor tandem, tumor cell migration is conducted (Neagu(b) et al., 2015). Moreover, adhesion of tumor cells to microvessel walls and extravasation into the target tissue is coordinated also by chemokines and their specific receptors (Kulbe et al, 2004, Ben-Baruch, 2008; Kawada et al., 2004). Invasion of melanoma cells into lymph node is favored by CCR7 (Murakami et al, 2004) CCR10, (Simonetti et al., 2006) CXCR3 and CXCR4 (Robledo et al, 2001) expression. Pulmonary melanoma metastasis is coordinated by CXCR4 expression while skin metastasis by CCR10 (Murakami et al, 2004). Deregulation of CXCR4/CXCL12 axis was found linked to BRAF mutation (Mitchell and Mahalingam M, 2014). In a transgenic mice model over-expressing CXCL14/BRAK when subjected to experimental melanoma, the animal's survival rates were found increased (Hata et al, 2015). CXCL14 induces the migration of activated NK cells (Starnes et al, 2006) and in melanoma patients it was shown that NK cell activation, actual cyto- 
toxic function and phenotype can control tumor development. We have shown that NK in melanoma-bearing mouse the percentage of NK cells in spleen, secondary lymph organ, is significantly reduced displaying different phenotypes compared to controls (Isvoranu et al., 2019).

CXCL8 (IL-8) has been found increased while CXCR1 was found down-regulated when melanoma cells were cultivated in suspension. The report shows that CXCL8 and CXCR expression is involved in the migration and metastasis processes (Uen et al., 2015). The circulatory levels of IL-8 can pinpoint the overall inflammatory status of a developing melanoma. We have shown in both animal models of melanoma (Surcel et al., 2017) and in melanoma patients

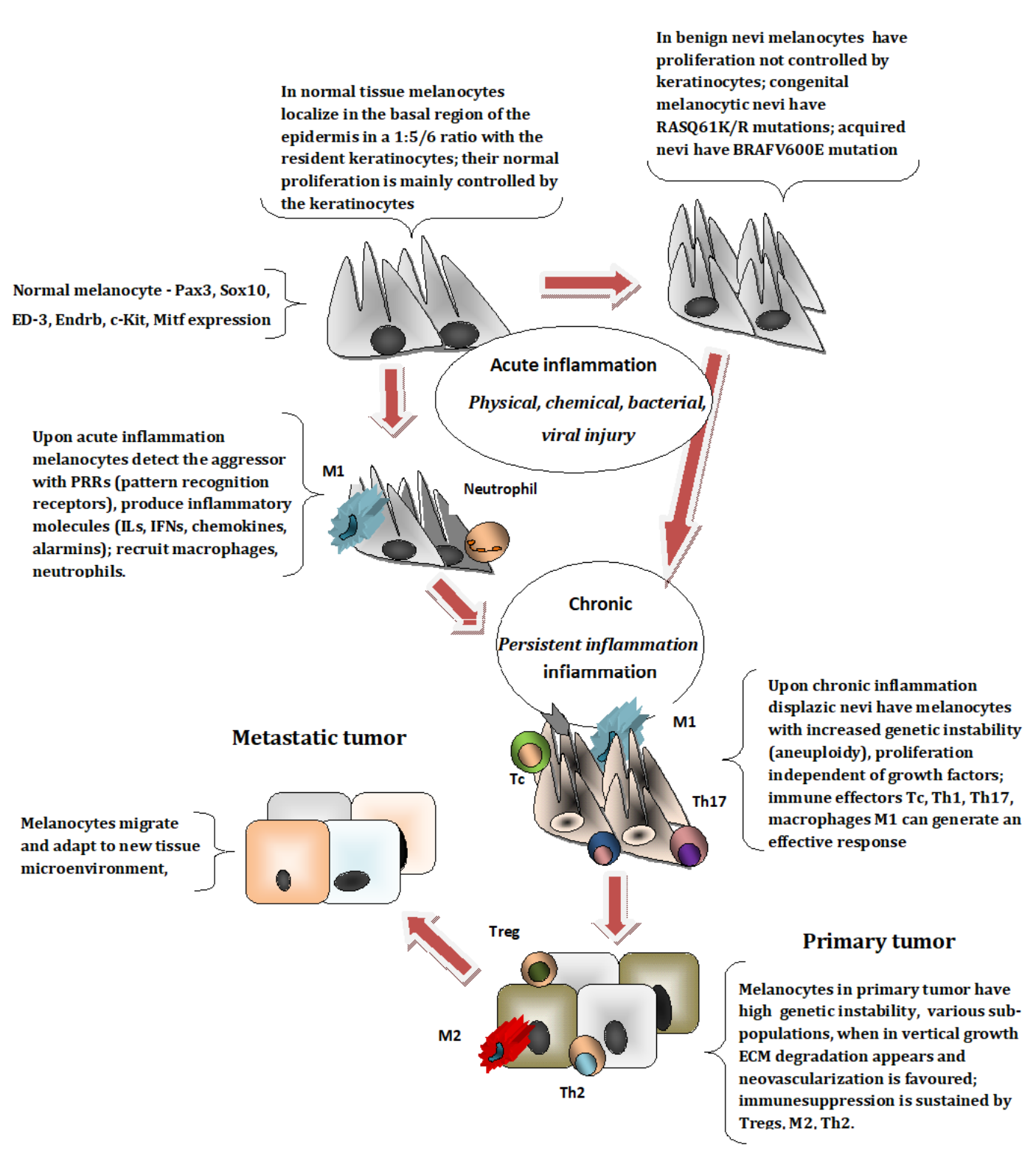

that IL-8 (Ene et al., 2015) was found significantly increased in melanoma bearing organisms correlated to the clinical evolution of the disease.

Chronic inflammation with all its array of cells, mechanisms and molecules can trigger and further sustain the pro-inflammatory processes leading to melanocyte neoplastic transformation. In Figure 1 a scheme of the main processes involved in the transformation of normal melanocyte towards a melanoma tumor is presented.

\section{Metabolic traits of melanomagenesis}

Within the patterns that govern melanomagenesis, recently, metabolic deregulations gained increased significance. By comparison,
Figure 1

In normal skin the melanocyte is characterized by Pax3, Sox10, ED-3, Endrb, c-Kit, Mitf expression. When un-damaged tissue is subjected to various bacterial, viral, chemical, physical injuries, an acute inflammation will induce melanocytes to detect the aggressor with PRRs (pattern recognition receptors), produce inflammatory molecules (ILs, IFNs, chemokines, alarmins); innate immunity cells (neutrophils and macrophages type $M_{1}$ ) will be recruited and will infiltrate the tissue to generate the tissue repair cascade. If the inflammation is sustained and has chronic characteristics a displazic nevi will have melanocytes with increased genetic instability (aneuploidy), proliferation independent of growth factors; immune effectors Tc, Th1, Th17, macrophages $M_{1}$ will infiltrate the tissue and generate an effective response and restore a normal status of the tissue; If the chronic inflammation persists then the pre-cancerous lesion turns into a full blown primary tumor. Melanocytes in primary tumor have high genetic instability, various subpopulations, and when the infiltrating cells are $M_{2}$ type macrophages Th2, Treg lymphocytes a protumorigenesis milieu is enhanced and the primary tumor turns into an aggressive one and starts to metastasize adapting to new microenvironments. 
a normal and a tumor cell have divergent metabolic status. The tumor cell has an altered metabolism and thus various different metabolic markers. The metabolic need is different in normal compared to tumor cell; the metabolism of a tumor cell has to be more dynamic, needs an increased metabolic flux and more nutritional factors. This metabolism is required to support an accelerated cell proliferation, to sustain migration, to survive in hypoxia conditions and to adapt to different tissue environments when engaged in metastasis.

Tumor cell metabolism would generate increased lactate production, nitric oxide (NO), reactive oxygen species (ROS) and arachidonic acid by-products (prostaglandins). All these molecules contribute to sustaining the inflammatory milieu and a tumor-permissive environment (Netea-Maier et al., 2018). The abnormal metabolism induces the expression of various dysfunctional proteins enhancing the pro-tumorigenic mechanisms. Therefore, tumor cell metabolism will lead to a deregulated cell cycle, enhanced anti-apoptotic cellular patterns, decreased cell death, increased migratory capacity and high adaptability to various non-related tissue microenvironment (Wang et al., 2018).

As in various other human cancers, in melanoma, glucose metabolism is the main deregulated metabolic cycle, leading to protein and gene deregulations (Gentric et al., 2017). As the main fuel controlling organelle, mitochondria, is highly involved in tumorigenesis through various pathways and metabolic alterations as decreased oxidative phosphorylation (OXPHOS) and anabolic pathways induction. Mitochondria controls ROS levels, DNA mutations, namely genomic instability, it controls autophagy and resistance to cell death stimuli (Masgras et al., 2017; Neagu(c) et al., 2019).

\section{Neuroendocrine "influencers" of melanomagenesis}

In the skin there is an array of neuroendocrine factors that are involved in various physiological and pathophysiological processes (Caruntu(a) et al., 2014). Moreover, these factors can influence also skin melanoma formation and progression, results obtained by several research teams, including our team (Colucci and Moretti, 2016, Caruntu(b) et al., 2014). Therefore, several reports have shown that catecholamines can induce melanoma progression and can stimulate melanoma cell proliferation through beta-adrenergic receptors activation (Janik et al., 2017, Sereni et al., 2015).

It seems that melanoma cells have different receptors expression and hence susceptibility to respond to adrenaline. Thus, depending on the origin and progression stage, metastatic skin melanoma cells are less responsive to adrenaline, while primary skin and uveal melanoma cells are more sensitive (Janik et al, 2017). We have shown on murine melanoma cell lines that exposure to high concentrations of epinephrine and norepinephrine induces a significant increase in cell proliferation (Caruntu(b) et al, 2014). There are different expression of specific receptors depending on the melanoma origin, thus in human melanoma cell lines we have shown that the proliferative action triggered by epinephrine and nor-epinephrine can be overridden by some of specific inhibitors (Surcel et al., 2018).

Recent data gather toward the clear influence of the neuroendocrine axis on the melanoma development and progression.

\section{Melanomagenesis}

Melanomagenesis is not a straight forward process, various factors collide, hence environmental, genetic, and immune factors must conjoint. Melanomas are actually a heterogeneous cells architecture; within the transformed melanocyte various genetic alterations occur. These mutations can be specific for the site where the tumor is developing, can be specific for exposed versus non-exposed to UV skin, can be specific to mucosa versus skin and so on. This high variability suggests that melanoma has more than one causal pathway. Thus, more than ten years ago, a molecular classification was proposed for melanomas based on the mutation panel, site of development and sun exposure history melanomas (Curtin et al., 2005). For example, BRAF mutations are more common in tumors without chronic sun exposure, while sun-non-exposed skin, mucosal surfaces, and acral skin melanomas have genetic alteration of KIT while lacking mutations in BRAF or NRAS. Out of all other risk factors in melanomagenesis, skin's phototype, high number of nevi/dysplasic nevi and familial history of melanoma are the clearest risk factors (Curtin et al, 2005). 
Mutational status identifies changes of nevi in the course of melanoma development, but there is still subject of debate if melanomas develop either de novo or upon neoplastic transformation of a naevus. Several years ago, when testing over 60 melanomas that have developed on a pre-existing naevi, interesting results were obtained. After selecting by micro-dissection the cells subpopulation the sequencing for BRAF, NRAS, C-KIT, PPP6C, STK19 and RAC 1 genes was performed. In almost $67 \%$ of the analyzed cases, a concordance of the mutational status of melanoma cells with naevi cells was obtained for all the investigated genes. Over $15 \%$ of all the investigated cases have had concomitant BRAF and NRAS mutations, moreover in one case types $\mathrm{V} 600 \mathrm{E}$ and $\mathrm{Q} 61 \mathrm{~K}$ double mutations were identified in both tissues, melanoma and nevi. This was actually the first large study that demonstrated melanomas and adjacent naevi sharing very similar mutational profile, and giving arguments that melanoma has a naevi origin (Shitara et al., 2015). Later, when studying Nevus of Ota that further developed in uveal-like dermal melanoma similar results were obtained. The Nevus of Ota contained GNAQ mutations, tumors that further developed had an increased RAS pathway expression. During tumorigenesis, whole-exome sequencing has shown that the lesions acquired BAP1 and TP53 mutations and that the actual tumor has an increased clonally heterogeneity (Vivancos et al., 2016).

Moving on to a clearly established melanoma, different genetic identity would characterize primary versus metastatic tumors. Using as clonality marker BRAF(V600E) mutation over 100 melanoma primaries and metastasis were studied. Mutant-specific PCR (MS-PCR) for BRAF along with conventional sequencing showed that in over one third of melanomas, mutations were identified using conventional sequencing, while over $75 \%$ using MS-PCR. Laser microdissection on specific cell subpopulations, mutation detection and BRAF(V60oE)-specific SNaPshot analysis showed that there are different percentages of BRAF(V60oE) mutations inside individual tumors. MS-PCR has shown that almost one third of patients that have melanoma metastases the genetic profile is not in accordance with their primary tumors' genetic status. As targeted therapy is used already in clinic, the response and/or the resistance to the therapy can be accounted for this tumor heterogeneity in terms of mutations (Yancovitz et al., 2012).

The last consensus in melanoma guidelines acknowledges that around $45 \%$ of patients with melanoma are carrying BRAF(V600) mutation and identifying mutational pattern in primaries versus metastasis would better guide targeted therapies (Garbea et al., 2019).

Melanoma cells are not genetically homogenous and moreover they can be in different stages of development. A recent report on melanoma cell lines and primary melanomas has shown that the gene expression analysis can sub-classify melanomas in four progressive subtypes. These types have specific sensitivity to ferroptosis and iron-dependent oxidative stress. Resistance to targeted therapies (e.g. anti-BRAF) and to immune therapy implies these transitions stages where ferroptosis sensitivity increases. Thus, in the process of resistance acquisition in melanoma, ferroptosis process can be also targeted to aim at the differentiation plasticity of melanoma cells (Tsoi et al. 2018).

The MAPK pathway controls cell proliferation, invasion, migration, and survival. In melanoma, the MAPK signaling pathways is constitutively activated due to membrane receptors alterations and/or through RAS or BRAF mutations. The mutated BRAF is in $90 \%$ of cases a valine to glutamic acid substitution (V600E), this mutation inducing constitutive kinase activation. $\mathrm{PI}_{3} \mathrm{~K}$ pathway activation, identified in melanoma is affecting various downstream effectors, e.g. AKT, mTOR, NF-kB, p53, and all these molecules are contributing to melanoma aggressiveness. Deregulation of $\mathrm{Pl}_{3} \mathrm{~K}$ activity can be achieved through loss of PTEN through mutation, gene deletion, or promoter methylation. p53 expression is associated with anti-tumor effects, thus restoration of $p_{53}$ function represents a good therapeutic goal that can be achieved alongside BRAF(V600E) inhibition (Lu et al., 2014).

Another molecule that characterizes a melanoma cell is melanocortin receptor $1\left(M C_{1} R\right)$; this receptor is involved in human pigmentation, UV response, and DNA damage. This is a melanocyte-specific $\mathrm{G}$ protein receptor that normally is involved in the physiology of the mela- 
nocyte and upon neoplastic transformation can be found deregulated.

A tyrosine receptor, KIT that links the stem cell factor (SCF) has an important role in melanocyte development. The tandem SCF-KIT stimulates MITF-M activity through post-translation phosphorylation (Yun et al., 2020). These molecules are related to clinical responses when therapy is applied, e.g. imatinib therapy targeting KIT mutations (Hodi et al., 2013).

In uveal melanomas $\mathrm{GNAQ} / 11^{\mathrm{Q} 209 \mathrm{~L}}$ mutations can induce pro-tumorigenic alteration within melanocytes, hyperpigmentation, altered cell migration, survival properties, evasion of normal boundary (Perez et al., 2018; Vultur and Herlyn, 2013; Hodis et al, 2012).

Melanoma antigen A (MAGE-A) protein is another marker for melanoma. These proteins are a sub-family of Cancer/Testis antigens involved in malignancy. MAGE-A regulates cancerrelated transcription factors, including $\mathrm{p} 53$, and is activating RING finger-dependent ubiquitin E3 ligases. It was shown that MAGE-A2 associates with a specific ubiquitin E3 ligase (MDM2), that ubiquitylates various substrates including $\mathrm{p}_{53}$, $M M_{2}$ itself, and MDM4. The reported data show that MAGE-A proteins expression on melanoma cells correlates to patient's clinical parameters (Marcar et al., 2015).

\section{Outline of high-throughput technologies involved in melanoma cellular patterns identification}

Without being exhaustive, we are giving a brief description of the recent technologies that are used to describe cellular portrait knowing that in the last 20 years, high-throughput approaches for studying genomes, proteomes, transcriptomes and metabolome components of melanoma cells were developing in an accelerated pace (Bulman et al, 2013).

Tumor initiation, development, and metastasis are complex, multi-factorial processes, therefore multi-omics technologies have been developed to cover these complex pathways, gathering humongous amount of data. It has been shown in melanoma that combining somatic mutation, DNA methylation and gene expression levels specific "somatic signatures" could be established. Molecular deregulation identified in cell-cycle regulation and in signal transduction pathways are the main traits of melanoma (Hua et al., 2016). In The Cancer Genome Atlas there are myriads of omics data and it was reported that for skin melanoma, multiintegration approach of these data can lead to prognostic models (Jiang et al., 2016). Actually, skin was among the first organs upon which complex genomic and proteomic analysis was done. Using these multi-omics technologies markers for disease prognosis and/or for therapies efficacy monitoring was identified. In this area array-based comparative genomic hybridization technology (Nambiar et al., 2008) can offer the possibility to analyze copy number variation (CNVs) within skin melanomas. This technology identifies new chromosomal alterations and discovers new deregulated melanoma genes that can be further used as therapy targets (Dumitru et al, 2017).

Another recent technology that can characterize complex cellular pattern is single-cell RNA sequencing (scRNA-seq). This technology characterizes patterns of both tumor cells and tumor-associated cell types. Using this technology to investigate heterogeneity of the cellular composition of various tumors astonishing details were furnished (Lavin et al., 2017; Tirosh et al., 2016; Zheng et al., 2017). Technologies that use single-cell sequencing data can show cellcell interrelation (Zhou et al., 2017), but the actual connection of the identified phenotypic features with biological outcomes in terms of both quantitative and qualitative measures are still limited. Recently a new technology was developed in order to identify cell-cell contact through ligand-receptor interactions using scRNA-seq data. In melanoma metastasis using this technology the association between singlecell and its microenvironment was studied. This new technology can be used to discover new therapeutic targets and new biomarkers that can be further used for patient stratification (Kumar et al, 2018).

In the quest to discover new cellular identities, single-cell transcriptomics can measure gene expression in each individual cell and multivariate analysis can show subtle expression variation during disease progression. Singlecell analysis can measure also transcriptome events and add information regarding molecular complexes that take place in intracellular pathways. Hence a cellular snapshot related to different temporal stages can be identified (Le- 
derer and La Manno, 2020).

Melanoma tumor cells are highly heterogeneous, for example when they have a higher expression of RUNX2 (osteogenic master gene) tumour progression and EMT processes are increased. Using CRISPR/Cas9 technology Deiana et al have shown in RUNT-deleted melanoma cell that these cells have reduced proliferation, increased apoptosis and decreased EMT traits. Using molecular technologies, it was depicted RUNX2 as a possible therapeutic target (Deiana et al., 2018).

Proteomic mass-spectrometry (MS) was put in use in several melanoma cell lines to evaluate the molecular events that hinder NK attack and favor EMT. Thus, using MS profiling it was shown that there is a proteomic common pattern related to the type of exposure, NK cells or EMT factors. Proteins that append to metabolic processes, to cell-cycle pathways are deregulated and authors suggest that "dormant tumor cells" can be generated through these NK-mediated mechanisms (Huergo-Zapico et al, 2018; Neagu(b) et al, 2019). MS technology was also put in use to demonstrate that during melanoma development several other non-melanoma cells have activated status and express and shed in circulation specific proteins like chemokines secreted by keratinocytes (Constantin et al., 2017).

In a recent study, several technologies were explored to evaluate the heterogeneous cellular profile of metastatic melanoma. Thus, multiplex immunohistochemistry (IHC) and flow cytometry (FACS) were used to quantify the immune populations that populate the tumor versus the ones that make-up the TME so that the tumor margins can be thoroughly depicted. It was shown that using multiplex IHC a correlation between $C D 8^{+} T$ cells infiltrating the tumor and PDL1 expression in melanoma was reported. Using FACS, T cell subset differentiation and the immune checkpoint molecules could be analyzed. Multiplex IHC identified more Tregs than FACS and more $\mathrm{CD}_{4}{ }^{+} \mathrm{T}$ cells in TME. Using these methods authors propose four categories of metastatic melanoma phenotypes:

presence/absence of $\mathrm{PDL} 1^{+}$on tumor cells and/or on macrophages, location in/out of the tumor, presence/absence of $\mathrm{CD} 8^{+} \mathrm{T}$ cells inside the tumor. These melanoma cellular sub-types can show a snapshot of the cellular heterogene- ity and further predict the therapeutical responses in an immune context (Halse et al. 2018).

In vivo FACS supplied with photoacoustic and fluorescence detectors can be used to identify circulating tumor cells (CTC). In melanoma, evaluating CTC is a test that seeks to evaluate as early as possible metastatic process installment and therapy efficacy (Jurati et al, 2014). The heterogeneity of melanoma cells was confirmed also using this test. Recently, using a combination of immunocytochemistry and transcript analyses of specific genes by RT-PCR and by droplet digital PCR (ddPCR), it was shown in the circulation of metastatic patients that are multiple non-overlapping tumor cell subpopulations (Aya-Bonilla et al., 2020).

\section{Conclusion}

Skin melanomas have a high degree of cellular heterogeneity due to various genetic alterations, these mutations being specific to the site, to the degree of UV exposure, and/or specific for the genetic make-up of the host's organism. The melanocyte changes its identity from normal melanocyte, through benign transformed melanocyte up to a full blown tumorigenesis. Various factors conjoin to these transformation, inflammatory, metabolic, neuroendocrine and many more probably unknown. Knowledge regarding the factors, the cooperation established between cells and the processes present in tumor microenvironment is still incomplete. Various omic's technologies should evolve in novel toolkits for investigation, where probably temporal-omics approaches can identify cellular patterns related to disease progression.

Integrating genetic profiling with all other types of proteomic/transcriptomic/metabolomic and correlating data with clinical and pathological parameters would lead to seminal improvements in diagnosis, prognosis and therapy in skin melanoma. 


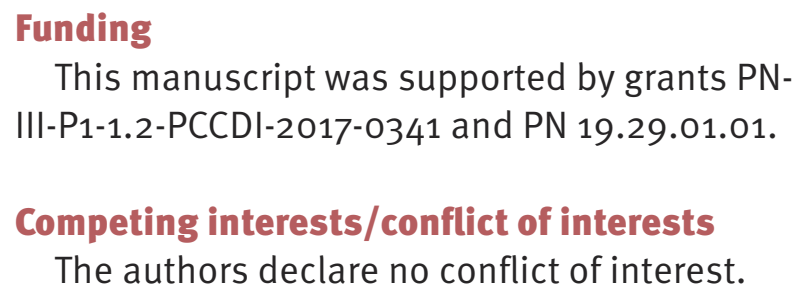

\section{References}

Abdel-Malek Z, Scott MC, Suzukietal I (2000) The melanocortin1 receptor is a key regulator of human cutaneous pigmentation. Pigment Cell Research 13(8): 156-162.

\section{Ackermann J, Frutschi M, Kaloulis K et al (2005)} Metastasizing melanoma formation caused by expression of activated N-RasQ61K on an INK4adeficient background. Cancer Research 65(10): 4005-4011.

\section{Arzumanyan A, Reis HM, Feitelson MA (2013)} Pathogenic mechanisms in HBV and HCVassociated hepatocellular carcinoma. Nat Rev Cancer 13:123-35. doi: 10.1038/nrc3449.

\section{Aya-Bonilla CA, Morici M, Hong X et al (2020)} Detection and prognostic role of heterogeneous populations of melanoma circulating tumour cells. Br J Cancer. doi: 10.1038/s41416-020-0750-9. [Epub ahead of print]

Barnes TA, Amir E (2017) HYPE or HOPE: The prognostic value of infiltrating immune cells in cancer. Br J Cancer 117: 451-460.

Ben-Baruch A (2008) Organ selectivity in metastasis: regulation by chemokines and their receptors. Clin Exp Metastasis 25(4):345-356.

\section{Bertolotto C, Abbe P, Hemesath TJ et al. (1998)} Microphthalmia gene product as a signal transducer in CAMP-induced differentiation of melanocytes. Journal of Cell Biology 142(3): 827835.

\section{Bertolotto C, Bille K, Ortonne J-P, Ballotti R (1996)} Regulation of tyrosinase gene expression by CAMP in B16 melanoma cells involves two CATGTG motifs surrounding the TATA box: implication of the microphthalmia gene product. Journal of Cell Biology 134(3): 747-755.
Bertolotto C, Busca R, Abbe P et al (1998) Different cis-acting elements are involved in the regulation of TRP1 and TRP2 promoter activities by cyclic AMP: pivotal role of $M$ boxes (GTCATGTGCT) and of microphthalmia. Molecular and Cellular Biology 18(2): 694-702.

Bertolotto C (2013) Melanoma: From Melanocyte to Genetic Alterations and Clinical Options, Scientifica Volume 2013, Article ID635203, 22 pages http://dx.doi.org/10.1155/2013/635203

\section{Bulman A, Neagu M, Constantin C (2013)} Immunomics in skin cancer - improvement in diagnosis, prognosis and therapy monitoring. Current Proteomics 10(3): $202-217$.

Busca R, Abbe P, Mantouxetal F (2000) Ras mediates the CAMP dependent activation of extracellular signal-regulated kinases (ERKs) in melanocytes. EMBO Journal 19(12): 2900-2910.

Caruntu(a) C, Boda D, Musat S, et al (2014) Stressinduced mast cell activation in glabrous and hairy skin. Mediators Inflamm.2014:105950. 9 pages.

Caruntu(b) C, Boda D, Constantin C, et al (2014) Catecholamines increase in vitro proliferation of murine B16F10 melanoma cells. Acta Endocrinologica (Buc) 10(4): 545-558.

\section{Castle PE, Hillier SL, Rabe LK, et al. (2001) An} association of cervical inflammation with highgrade cervical neoplasia in women infected with oncogenic human papillomavirus (HPV). Cancer Epidemiol Biomarkers Prev. 10: 1021-7.

Chiaverini C, Beuret L, Flori E et al (2008) Microphthalmia associated transcription factor regulates RAB27A gene expression and controls melanosome transport. Journal of Biological Chemistry 283(18): 12635-12642. 
Colucci R, Moretti S (2016) The role of stress and betaadrenergic system in melanoma: current knowledge and possible therapeutic options. J Cancer Res Clin Oncol.142(5):1021-9.

\section{Constantin C, Bulman A, McCarthy D, Neagu M (2017)} Surface-Enhanced Laser Desorption/Ionization Mass Spectrometry for Biomarker Discovery in Cutaneous Melanoma. Current Proteomics 14 (2): 100-111.

Cui R, Widlund HR, Feigeetal E (2007) Central role of p53 in the suntan response and pathologic hyperpigmentation. Cell 128(5): 853-864.

\section{Curtin JA, Fridlyand J, Kageshita T et al (2005)} Distinct sets of genetic alterations in melanoma. New England Journal of Medicine 353(20): 21352147.

Dadras SS, (2011) Molecular diagnostics in Melanoma: current status and perspectives," Archives of Pathology and Laboratory Medicine 135(7): 860-869.

\section{Damsky WE, Curley DP, Santhanakrishnan M et al.} (2011) catenin signaling controls metastasis in braf-activated ptendeficient melanomas Cancer Cell 20(6): 741-754.

Dankort D, Curley DP, Cartlidge RA et al., (2009) Braf V600E cooperates with Pten loss to induce metastatic melanoma," Nature Genetics 41(5): 544-552.

Deiana M, Dalle Carbonare L, Serena M et al (2018) New Insights into the Runt Domain of RUNX2 in Melanoma Cell Proliferation and Migration. Cells 7(11). pii: E220. doi: 10.3390/cells7110220

\section{Dempke WCM, Fenchel K, Uciechowski P, et al. (2017)} Second-and third-generation drugs for immunooncology treatment-The more the better? Eur. J. Cancer 74: 55-72.

\section{Denoyelle C, Abou-Rjaily G, Bezrookove V et al.} (2006) Antioncogenic role of the endoplasmic reticulum differentially activated by mutations in the MAPK pathway. Nature Cell Biology 8(10): 1053-1063.
Dhomen N, Reis-Filho JS, da Rocha Dias S et al. (2009) Oncogenic braf induces melanocyte senescence and melanoma in mice. Cancer Cell 15(4): 294-303.

Dumitru C, Constantin C, Popp C et al (2017) Innovative array-based assay for omics pattern in melanoma. Journal of Immunoassay and Immunochemistry 38(4): 343-354. http://dx.doi.org/10.1080/15321819.2017.134089 8

Dunn GP, Old LJ, Schreiber RD (2004) The immunobiology of cancer immunosurveillance and immunoediting. Immunity 21:137-48. doi: 10.1016/j.immuni.2004.07.017

Dynek JN, Chan SM, Liu J, et al (2008) Microphthalmia-associated transcription factor is a critical transcriptional regulator of melanoma inhibitor of apoptosis in melanomas. Cancer Research 68(9): 3124-3132.

Ene C-D, Anghel A-E, Neagu M, Nicolae I (2015) 25-OH Vitamin D and Interleukin-8 Emerging Biomarkers in Cutaneous Melanoma Development and Progression, Mediators of Inflammation, pages 18, Article ID 904876.

\section{Gaggioli C, Busca R, Abbe P et al (2003)} Microphthalmia-associated transcription factor (MITF) is required but is not sufficient to induce the expression of melanogenic genes. Pigment Cell Research 16(4): 374-382.

Gajewski TF, Corrales L, Williams J, et al (2017) Cancer immunotherapy targets based on understanding the T cell-inflamed versus non-T cell-inflamed tumor microenvironment. Adv Exp Med Biol. 1036:19-31. doi: 10.1007/978-3-319-67577-0_2

Garbe C, Amaral T, Peris K et al (2019) European consensus-based interdisciplinary guideline for melanoma. Part 2: Treatment - Update 2019, European Journal of Cancer, https://doi.org/10.1016/j.ejca.2019.11.015

\section{Gentric G, Mieulet V, Mechta-Grigoriou F (2017)} Heterogeneity in cancer metabolism: new concepts in an old field. Antioxid Redox Signal 26:462-85. doi: 10.1089 /ars.2016.6750 
Gonda TA, Tu S, Wang TC (2009) Chronic inflammation, the tumor microenvironment and carcinogenesis. Cell Cycle 8:2005-2013.

Halse H, Colebatch AJ, Petrone P, et al (2018) Multiplex immunohistochemistry accurately defines the immune context of metastatic melanoma. Sci Rep. 8(1):11158. doi: 10.1038/s41598-018-28944-3.

Hanahan D, Weinberg RA (2011). Hallmarks of cancer: the next generation. Cell 144: 646-674.

Haqa R, Yokoyamab S, Hawryluk E B et al. (2013) $B C L 2 A 1$ is a lineage-specific anti apoptotic melanoma oncogene that confers resistance to BRAF inhibition. Proc Natl Acad Sci USA 110(11): 4321-4326.

Hata R, Izukuri K, Kato Y, et al (2015) Suppressed rate of carcinogenesis and decreases in tumour volume and lung metastasis in CXCL14/BRAK transgenic mice. Sci Rep. 5:9083.

Hirobe T (2005) Role of keratinocyte-derived factors involved in regulating the proliferation and differentiation of mammalian epidermal melanocytes. Pigment Cell Research 18(1): 2-12.

Hodi FS, Corless CL, Giobbie-Hurder A et al (2013) Imatinib for Melanomas Harboring Mutationally Activated or Amplified KIT Arising on Mucosal, Acral, and Chronically Sun-Damaged Skin, Journal of Clinical Oncology 31(26): 3182-3194

Hodis E, Watson IR, Kryukov GV et al (2012) A landscape of driver mutations in melanoma. Cell 150: 251-263.

Hoek KS, Schlegel NC, Eichhoff OM et al (2008) Novel MITF targets identified using a two-step DNA microarray strategy. Pigment Cell \& Melanoma Research 21(6): 665-676.

Houghton AM (2013) Mechanistic links between COPD and lung cancer. Nat Rev Cancer. 13:233-45. doi: $10.1038 /$ nrc3477.

Hua L, Zheng WY, Xia H, Zhou P (2016) Detecting the potential cancer association or metastasis by multiomics data analysis. Genet Mol Res. 15 (3): 1-15
Huergo-Zapico L, Parodi M, Cantoni C, et al. (2018)

NK-cell Editing Mediates Epithelial-to-

Mesenchymal Transition via Phenotypic and Proteomic Changes in Melanoma Cell Lines. Cancer Res. 78(14):3913-3925. doi: 10.1158/00085472.CAN-17-1891

Isvoranu G, Surcel M, Huică R-I et al (2019) Natural killer cells monitoring in cutaneous melanoma new dynamic biomarker. Oncol Lett 17: 4197-4206.

Janik ME, Szlęzak D, Surman M, et al (2017) Diversified $\beta$-2-adrenergic Receptor Expression and Action in Melanoma Cells. Anticancer Res.37(6):3025-3033.

Jiang Y, Shi X, Zhao Q et al (2016) Integrated analysis of multidimensional omics data on cutaneous melanoma prognosis. Genomics 107 (6): 223-230.

Jimenez-Sanchez A, Memon D, Pourpe S, et al. (2017) Heterogeneous Tumor-Immune Microenvironments among Differentially Growing Metastases in an Ovarian Cancer Patient. Cell 170: 927-938.e20.

Juratli MA, Sarimollaoglu M, Nedosekin DA, et al (2014) Dynamic Fluctuation of Circulating Tumor Cells during Cancer Progression. Cancers (Basel). 6(1): 128-42. doi: 10.3390/cancers6010128

Karlou M, Tzelepi V, Efstathiou E (2010) Therapeutic targeting of the prostate cancer microenvironment. Nat Rev Urol. 7:494-509. doi: 10.1038 /nrurol.2010.134

\section{Kawada K, Sonoshita M, Sakashita H, et al (2004)} Pivotal role of $\mathrm{CXCR}_{3}$ in melanoma cell metastasis to lymph nodes. Cancer Res. 64(11):4010-4017.

Kulbe H, Levinson NR, Balkwill F, Wilson JL (2004) The chemokine network in cancer-much more than directing cell movement. Int J Dev Biol. 48(56):489-496.

Kumar MP, Du J, Lagoudas G, et al (2018) Analysis of Single-Cell RNA-Seq Identifies Cell-Cell Communication Associated with Tumor Characteristics. Cell Reports 25: 1458-1468.

Lavin Y, Kobayashi S, Leader A et al. (2017). Innate Immune Landscape in Early Lung Adenocarcinoma by Paired Single-Cell Analyses. Cell 169: 750-765.e17 
Lederer AR, La Manno G (2020) The emergence and promise of single-cell temporal-omics approaches. Curr Opin Biotechnol. 63:70-78. doi: 10.1016/j.copbio.2019.12.005.

Longo-Imedio MI, Longo N, Trevino I, et al (2005) Clinical significance of $\mathrm{CXCR}_{3}$ and $\mathrm{CXCR}_{4}$ expression in primary melanoma. Int J Cancer $117(5): 861-865$

Lu M, Miller P, Lu X (2014) Restoring the tumour suppressive function of p53 as a parallel strategy in melanoma therapy. FEBS Lett. 588(16): 2616-21. doi: 10.1016/j.febslet.2014.05.008.

Marcar L, Ihrig B, Hourihan J et al (2015) MAGE-A Cancer/Testis Antigens Inhibit MDM2 Ubiquitylation Function and Promote Increased Levels of MDM4. PLoS One. 10(5):e0127713. doi: 10.1371/journal.pone.0127713.

\section{Masgras I, Sanchez-Martin C, Colombo G, Rasola A}

(2017) The chaperone TRAP1 as a modulator of the mitochondrial adaptations in cancer cells. Front Oncol. 7:58. doi: 10.3389/fonc.2017.00058

\section{McGill GG, Horstmann M, Widlund HR et al. (2002)}

$\mathrm{Bcl} 2$ regulation by the melanocyte master regulator Mitf modulates lineage survival and melanoma cell viability Cell 109(6): 707-718.

\section{McGranahan N, Swanton C (2017). Clonal}

Heterogeneity and Tumor Evolution: Past, Present, and the Future. Cell 168: 613-628.

\section{Michaloglou C, Vredeveld LCW, Soengas MS et al} (2005) BRAFE600-associated senescence-like cell cycle arrest of humannaevi. Nature 436(7051) 720724.

Mitchell B, Mahalingam M (2014) The CXCR4/CXCL12 axis in cutaneous malignancies with an emphasis on melanoma. Histol Histopathol. 29(12):1539-46.

\section{Monteagudo C, Martin JM, Jorda E, Llombart-Bosch A} (2007) CXCR3 chemokine receptor immunoreactivity in primary cutaneous malignant melanoma: correlation with clinicopathological prognostic factors. J Clin Pathol, 60(6):596-599.
Murakami T, Cardones AR, Hwang ST (2004)

Chemokine receptors and melanoma metastasis. J Dermatol Sci 36(2):71-78.

\section{Nambiar S, Mirmohammadsadegh A, Hengge UR}

(2008) Cutaneous melanoma: fishing with chips. Curr Mol Med. 8(3): 235-243.

Neagu M (2012) The immune system - a hidden treasure for biomarker discovery in cutaneous melanoma, Adv Clin Chem, 58 : 89-140 ISBN: 9780-12-394383-5

Neagu M, Constantin C, Zurac S (2013) Immune parameters in prognosis and therapy monitoring of cutaneous melanoma patients - experience, role and limitations, BioMed Research International, special issue Molecular Biomarkers: Tools of Medicine (MBTM) 2013: Article ID 107940

Neagu M(a), Constantin C, Dumitrascu GR, Lupu AR et al (2015) Inflammation markers in cutaneous melanoma - edgy biomarkers for prognosis. Discoveries 3(1): e38114

Neagu(b) M, Constantin C, Longo C (2015) Chemokines in the Melanoma Metastasis Biomarkers Portrait. J Immunoassay Immunochem. 36(6):559-66.

Neagu(a) M, Constantin C, Caruntu C et al (2019) Inflammation - key process in skin tumorigenesis, Oncol Lett 17: 4068-4084. https://doi.org/10.3892/ol.2018.9735

Neagu(b) M, Constantin C, Bostan M et al (2019) Proteomic Technology “Lens” for EpithelialMesenchymal Transition Process Identification in Oncology. Anal. Cell. Pathol. (Amsterdam) 2019:117, 2019 Oct 29;2019:3565970. doi: $10.1155 / 2019 / 3565970$.

Neagu(c) M, Constantin C, Popescu ID et al (2019) Inflammation and metabolism in cancer cell mitochondria key player, Front Oncol. 9:348, doi: $10.3389 /$ fonc. 2019.00348

\section{Netea-Maier RT, Smit JWA, Netea MG (2018)}

Metabolic changes in tumor cells and tumorassociated macrophages: a mutual relationship. Cancer Lett. 413: 102-9. doi: 10.1016/j.canlet.2017.10.037 
Pardoll DM (2012). The blockade of immune checkpoints in cancer immunotherapy. Nat. Rev. Cancer 12: 252-264.

\section{Passeron T, Bahadoran P, Bertolotto C et al. (2004)} Cyclic AMP promotes a peripheral distribution of melanosomes and stimulates melanophilin/Slac2aactin association. FASEB Journal 18(9): 989-991.

Perez DE, Henle AM, Amsterdam A, et al (2018) Uveal melanoma driver mutations in $\mathrm{GNAQ} / 11$ yield numerous changes in melanocyte biology. Pigment Cell Melanoma Res. 31(5):604-613. doi: 10.1111/pcmr.12700.

Ramilowski JA, Goldberg T, Harshbarger J, et al. (2015). A draft network of ligand-receptormediated multicellular signalling in human. Nat. Commun. 6: 7866.

Raval RR, Sharabi AB, Walker AJ, et al (2014) Tumor immunology and cancer immunotherapy: summary of the 2013 SITC primer. J Immunother Cancer 2:14. doi: 10.1186/2051-1426-2-14

Robledo MM, Bartolome RA, Longo N, et al (2001) Expression of functional chemokine receptors CXCR3 and CXCR4 on human melanoma cells. I Biol Chem. 276(48):45098-45105.

Rothenberg SM, Ellisen LW (2012) The molecular pathogenesis of head and neck squamous cell carcinoma. J Clin Investig. 122:1951-7. doi: $10.1172 / \mathrm{JCl}_{59889}$

Salama NR, Hartung ML, Muller A (2013) Life in the human stomach: persistence strategies of the bacterial pathogen Helicobacter pylori. Nat Rev Microbiol. 11:385-99. doi: 10.1038/nrmicro3016

\section{Sarkar S, Sabhachandani P, Stroopinsky D, et al} (2016). Dynamic analysis of immune and cancer cell interactions at single cell level in microfluidic droplets. Biomicrofluidics 10: 054115.

\section{Schumacher TN, Kesmir C, van Buuren MM (2015)} Biomarkers in cancer immunotherapy. Cancer Cell 27: $12-14$.
Sereni F, Dal Monte M, Filippi L, Bagnoli P (2015) Role of host $\beta 1$ - and $\beta 2$-adrenergic receptors in a murine model of B16 melanoma: functional involvement of $\beta 3$-adrenergic receptors. Naunyn Schmiedebergs Arch Pharmacol 388(12):1317-31.

Shitara D, Tell-Martí G, Badenas C, et al (2015) Mutational status of naevus-associated melanomas. $\mathrm{Br}$ J Dermatol. 173(3):671-80. doi: 10.1111/bjd.13829.

Simonetti 0, Goteri G, Lucarini G, et al (2006) Potential role of CCL27 and CCR10 expression in melanoma progression and immune escape. Eur J Cancer 42(8):1181-1187.

Spranger S (2011) Mechanisms of tumor escape in the context of the T-cell-inflamed and the non-T-cellinflamed tumor microenvironment. Int Immunol. 28(8):383-91. doi: 10.1093/intimm/dxw014.

Starnes, T. Rasila KK, Robertson MJ, et al (2006) The chemokine CXCL14 (BRAK) stimulates activated NK cell migration: implications for the downregulation of CXCL14 in malignancy. Exptl Hematol 34, 11011105 .

Strub T, Giuliano S, Ye T et al. (2011) Essential role of microphthalmia transcription factor for DNA replication, mitosis and genomic stability in melanoma. Oncogene 30(20): 2319-2332.

Surcel M, Căruntu C, Tampa M et al (2018) Adrenergic Modulation of Melanoma Cells Proliferation. Farmacia 66(5): 820-825.

\section{Surcel M, Constantin C, Caruntu C, et al (2017)} Inflammatory cytokine pattern is sex-dependent in mouse cutaneous melanoma experimental model, Journal of Immunology Research, 2017, Article ID 9212134, 10 pages, https://doi.org/10.1155/2017/9212134

Tirosh I, Izar B, Prakadan SM et al. (2016) Dissecting the multicellular ecosystem of metastatic melanoma by single-cell RNA-seq. Science 352: 189-196.

Tsoi J, Robert L, Paraiso K, et al (2018) Multi-stage Differentiation Defines Melanoma Subtypes with Differential Vulnerability to Drug-Induced IronDependent Oxidative Stress. Cancer Cell 33(5):890-904.e5. doi: 10.1016/j.ccell.2018.03.017. 
Tzanakakis GN, Neagu M, Tsatsakis AM, Nikitovic D (2019) Proteoglycans and immunobiology of cancer - therapeutic implications, Frontiers in Immunology, section Cancer Immunity and Immunotherapy 10, Article 875 doi: 10.3389/fimmu.2019.00875

Uen WC, Hsieh CH, Tseng TT, et al (2015) Anchorage independency promoted tumor malignancy of melanoma cells under reattachment through elevated interleukin- 8 and CXC chemokine receptor 1 expression. Melanoma Res. 25(1):35-46.

Van Schanke A, Jongsma MJ, Bisschop R et al (2005) Single UVB overexposure stimulates melanocyte proliferation in murine skin, in contrast to fractionated or UVA-1 exposure. Journal of Investigative Dermatology 124(1): 241-247.

Vetrini F, Auricchio A, Du J et al (2004) The microphthalmia transcription factor (Mitf) controls expression of the ocular albinism type 1 gene: link between melanin synthesis and melanosome biogenesis. Molecular and Cellular Biology 24(15): 6550-6559.

Vinay DS, Ryan EP, Pawelec G et al. (2015) Immune evasion in cancer: mechanistic basis and therapeutic strategies. Semin Cancer Biol. 35(Suppl.) S185-98. doi: 10.1016/j.semcancer.2015.03.004

Vivancos A, Caratú G, Matito J et al. (2016) Genetic evolution of nevus of Ota reveals clonal heterogeneity acquiring $\mathrm{BAP}_{1}$ and $\mathrm{TP}_{53}$ mutations. Pigment Cell Melanoma Res. 29(2):247-53. doi: 10.1111/pcmr.12452.

Vultur A, Herlyn M (2013) SnapShot: Melanoma. Cancer Cell 23: 706-706.e1. DOI http://dx.doi.org/10.1016/j.ccr.2013.05.001.

Walker GJ, Kimlin MG, Hacker E et al. (2009) Murine neonatal melanocytes exhibit a heightened proliferative response to ultraviolet radiation and migrate to the epidermal basal layer. Journal of Investigative Dermatology 129(1): 184-193.
Wang Z, Wang N, Zheng Y, Wang S (2018)

Inflammasome and Cancer. Exp Suppl. 108:281302. doi: 10.1007/978-3-319-89390-7_12

Weiss SA, Han SW, Lui K, et al (2016) Immunologic heterogeneity of tumor-infiltrating lymphocyte composition in primary melanoma. Hum Pathol 57: 116-125.

Whiteman DC, Pavan WJ, Bastian BC (2011) The melanomas: a synthesis of epidemiological, clinical, histopathological, genetic, and biological aspects, supporting distinct subtypes, causal pathways, and cells of origin. Pigment Cell \& Melanoma Research 24(5): 879-897.

Yancovitz M, Litterman A, Yoon J, et al. (2012) Intraand inter-tumor heterogeneity of BRAF(V600E)) mutations in primary and metastatic melanoma. PLoS One 7(1):e29336. doi: 10.1371/journal.pone.0029336.

Yun CY, Roh E, Kim SH et al (2020) Stem Cell FactorInducible MITF-M Expression in Therapeutics for Acquired Skin Hyperpigmentation. Theranostics. 10(1):340-352. doi: 10.7150/thno.39066.

Zheng C, Zheng L, Yoo JK et al (2017) Landscape of Infiltrating T Cells in Liver Cancer Revealed by Single-Cell Sequencing. Cell 169: 1342-1356.e16

Zhou JX, Taramelli R, Pedrini E (2017) Extracting Intercellular Signaling Network of Cancer Tissues using Ligand-Receptor Expression Patterns from Whole-tumor and Single-cell Transcriptomes. Sci. Rep. 7, 8815

Zurac S, Negroiu G, Andrei R, et al (2013) Inflammatory infiltrate in melanoma with regression as prognostic parameter. Virchows Arch 463: 127. 
Celli İdentity 